\title{
Time-domain hybrid method for simulating large amplitude motions of ships advancing in waves
}

\author{
Shukui Liu and Apostolos D. Papanikolaou
}

Ship Design Laboratory, National Technical University of Athens, Athens, Greece

\begin{abstract}
Typical results obtained by a newly developed, nonlinear time domain hybrid method for simulating large amplitude motions of ships advancing with constant forward speed in waves are presented. The method is hybrid in the way of combining a time-domain transient Green function method and a Rankine source method. The present approach employs a simple double integration algorithm with respect to time to simulate the free-surface boundary condition. During the simulation, the diffraction and radiation forces are computed by pressure integration over the mean wetted surface, whereas the incident wave and hydrostatic restoring forces/moments are calculated on the instantaneously wetted surface of the hull. Typical numerical results of application of the method to the seakeeping performance of a standard containership, namely the ITTC S175, are herein presented. Comparisons have been made between the results from the present method, the frequency domain $3 D$ panel method (NEWDRIFT) of NTUA-SDL and available experimental data and good agreement has been observed for all studied cases between the results of the present method and comparable other data.
\end{abstract}

KEY WORDS: Hybrid time domain method; Nonlinear ship motion; Chimera grid; Free surface simulation.

\section{INTRODUCTION}

In recent years, the importance of a reliable prediction of the seakeeping behavior of ships in heavy seas has significantly increased, as it affects both the design and operation of ships. For about six decades, the seakeeping behavior of ships has been extensively studied by linear quasi two dimensional, strip or slender body theory or 3D panel, frequency domain methods. More recently, however, nonlinear time domain methods are more and more displacing linear methods, as they enable the address of large amplitude motion problems, which is very important for the design and safe operation of modern ships, operating in a variety of adverse environmental conditions.

Following the pioneering work of Finkelstein (1957) and Cummins (1962), many researchers investigated seakeeping problems by different time domain approaches and showed promising results for both linear and nonlinear problems of different level. In line with Lin's work (Lin et al., 1990), the authors proceeded with the development of a Transient Green Function method and demonstrated its implementation by application to some fundamental nonlinear problems (Liu et al., 2006). But this method proved not satisfactory in some practical cases. When it is applied to floating bodies with a

Corresponding author: Apostolos D. Papanikolaou e-mail:papa@deslab.ntua.gr flare, which is very common to modern ship designs, numerical problems may arise and computations are disturbed. Duan et al. (1999) found that the commonly used panel method using transient Green function for a non-wallsided floating body does not satisfy the mean-value theorem of definite integrals for the near water surface panels and solved this problem by introducing an imaginary vertical surface, which encloses the hull surface in the fluid domain. This method works fine, unless the body has some bulb-like sectional form, which exceeds the projection of the water plane.

Likewise the present hybrid method, Zhang et al. (1998) decomposed the fluid domain into two parts by a matching cylindrical surface, which is at some distance away from the body and is moving at the same speed as the ship; two potential theory boundary value problems were set-up and solved for the inner and outer domain potential in the time domain. Following the above work, Yasukawa (2003) and Kataoka et al. $(2004,2005)$ used similar schemes to solve the problem.

In line with above works, in this paper, a nonlinear time domain hybrid method for simulating motions of ships in waves with forward speed is presented. The fluid domain is decomposed into an inner and an outer part. The Rankine source method is applied in the inner domain to find the dominant equation, while the transient Green function method is used in the outer domain to obtain a relationship 
between the velocity potential and its normal derivative on each panel, which forms a boundary condition for the inner domain solution. This hybrid method works efficiently with a relatively small number of panels, for the free surface panelization is restricted between the body boundary and the control surface. Unlike above mentioned similar approaches, a simple double integration algorithm with respect to time, originally developed by Wang (2003), is herein adopted to simulate the free-surface boundary condition.

In order to validate the theoretical method and numerical scheme (computer code), some characteristic studies on the hydrodynamic forces and motions of standard type ships have been conducted. Results are compared with those of other authors and available experimental data. The major outcome of the presented method is its successful application to the assessment of the ITTC standard ship S175, which has nonwall-sided sections at the ends, while moving with forward speed in head seas. Good agreement has been observed for all studied cases between the results of the present method, other numerical codes and experimental data. Thus, the method proves promising regarding its applicability to the more general large amplitude motion problem of ships, advancing in waves of arbitrary heading.

\section{FORMULATION}

Consider a 3D body floating on the free water surface, advancing at constant forward speed and undergoing 6-DOF motions in response to an incident wave. An earth-fixed Cartesian coordinate system $O-x y z$ is chosen with the $x-y$ plane coincident with the undisturbed free surface and z-axis pointing upwards through the ship's mass centroid, as shown in Fig 1. The fluid is assumed to be idealized and the water depth infinite. The ship's forward speed $U_{0}$ is in $x$-axis direction.

Potential theory is used to determine the flow field. First, the flow field is decomposed into two parts, i.e. the inner domain denoted as $I$, bounded by the wetted body surface $S_{b}$, the cylindrical control surface $S_{c}$ and a part of the free surface $S_{f}$, namely between $S_{b}$ and $S_{c}$; and the outer domain denoted as $I I$, enclosed by the control surface $S_{c}$, the remaining free surface and the boundary surface at infinity (Fig. 1).

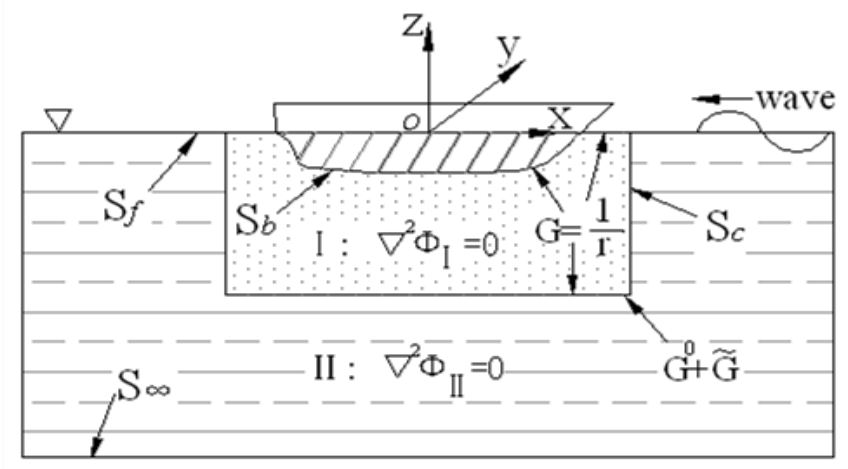

Fig. 1 Coordinate system.

\section{Integral equations}

In the inner domain $I$, the Rankine source method is used to determine the potential of the flow field. The integral equation takes the form of:

$\iint_{S_{c}+S_{b}+S_{f}}\left[\Phi_{I}(q, t) \frac{\partial}{\partial n_{q}}\left(\frac{1}{r_{p q}}\right)-\frac{1}{r_{p q}} \frac{\partial}{\partial n_{q}} \Phi_{I}(q, t)\right] d s_{q}=-4 \pi \Phi_{I}(p, t)$

where $\mathbf{n}$ is the unit normal vector pointing outward of the inner domain.

Let the field point $p$ approach the inner domain boundary, then we will get:

$$
\begin{array}{r}
\iint_{S_{c}+S_{b}+S_{f}}\left[\Phi_{I}(q, t) \frac{\partial}{\partial n_{q}}\left(\frac{1}{r_{p q}}\right)-\frac{1}{r_{p q}} \frac{\partial}{\partial n_{q}} \Phi_{I}(q, t)\right] d s_{q}=-2 \pi \Phi_{I}(p, t) \\
\left(p \in S_{I}\right)
\end{array}
$$

where $q(\xi, \eta, \zeta)$ is the source point, $p(\mathrm{x}, \mathrm{y}, \mathrm{z})$ is the field point; $\mathbf{r}_{\mathrm{pq}}=(\mathrm{x}-\xi) \mathbf{i}+(\mathrm{y}-\eta) \mathbf{j}+(\mathrm{z}-\zeta) \mathbf{k}$.

In the outer domain II, the transient time-domain Green function is employed to determine the disturbed potential on the control surface $S_{c}$. The relevant integral equation is expressed as:

$$
\begin{aligned}
2 \pi \Phi_{I I}(p, t) & +\iint_{S_{c}}\left[\Phi_{I I}(q, t) \frac{\partial}{\partial n_{q}}\left(\frac{1}{r_{p q}}-\frac{1}{r_{p q}}\right)-\left(\frac{1}{r_{p q}}-\frac{1}{r_{p q}}\right) \frac{\partial \Phi_{I I}}{\partial n_{q}}\right] d S_{q} \\
& =\int_{0}^{t} d \tau \iint_{S_{c}}\left[\tilde{G} \frac{\partial \Phi_{I I}}{\partial n_{q}}-\Phi_{I I} \frac{\partial \tilde{G}}{\partial n_{q}}\right) d s_{q} \quad\left(p \in S_{c}\right)
\end{aligned}
$$

On the control surface, the potential solutions from the inner domain model and the outer domain model should match each other. Considering the definitions of the normal vectors in different domains we have:

$\Phi_{I}=\Phi_{I I}, \frac{\partial \Phi_{I}}{\partial n}=-\frac{\partial \Phi_{I I}}{\partial n} \quad\left(\right.$ on $\left.S_{c}\right)$

The velocity potential in the inner domain can also be expressed by a pulsating simple source distribution, namely as:

$$
4 \pi \Phi(p, t)=\iint_{S_{c}+S_{b}+S_{f}} \sigma(q, t) \frac{1}{r_{p q}} d s_{q}
$$

Having obtained $\Phi$ through the aforementioned hybrid solver, the source density $\sigma$ can be evaluated by solution of the integral Equation (4). Following this, the spatial derivatives can be evaluated as:

$$
\frac{\partial \Phi(p, t)}{\partial x_{k}}=\frac{1}{4 \pi} \int_{S_{c}+S_{b}+S_{f}} \sigma(q, t) \frac{\partial}{\partial x_{k}} \frac{1}{r_{p q}} d s_{q}
$$




\section{Free-surface condition}

The linearized free-surface condition can be expressed in this coordinate system as:

$\frac{\partial^{2} \Phi}{\partial t^{2}}+g \frac{\partial \Phi}{\partial z}=0$

Integrating the above equation with respect to time $t$ twice and taking into account the initial conditions, we will get the following expression:

$\Phi=-g \int_{0}^{t}(t-\tau) \frac{\partial \Phi(p, \tau)}{\partial n} d \tau$

This formulation, first adopted by Wang (2003), is simple and also proved to be a robust free-surface numerical simulator (Liu et al., 2009). It should be noted in relation to the development and implementation of the above expression, that the integration with respect to time is from moment 0 to moment $t$ and the initial condition is $\left.\operatorname{set} \Phi\right|_{t=0}=\left.\frac{\partial \Phi}{\partial t}\right|_{t=0}=0$. This integral makes of course sense only for the wetted free surface area, which is not occupied by the advancing ship hull. Some special attention and treatment of the free surface boundary condition and corresponding panels is needed for the part of the free surface area, which is crossed and for some time occupied by the advancing ship hull (Fig. 2).

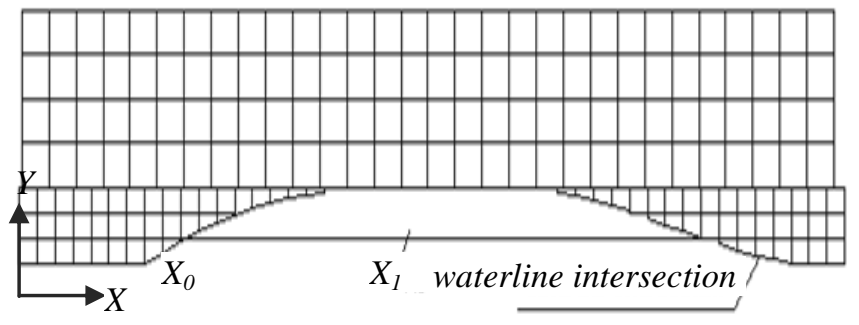

Fig. 2 Ship crossing free surface panels.

As shown in the above graph, if the intersection point between the ship's waterline WL and some $y=$ const is $x=x_{0}$ at moment $t_{0}$, then for a point at $x=x_{1}$ which is occupied by the hull at the moment, it will take some time, $\left(x_{1}-x_{0}\right) / U_{0}$, for this point to become active (wetted). The treatment of a free surface panel at this point is as following:

1) Do the $1^{\text {st }}$ integration of the expression regarding to time $\tau \in\left(t_{0}, t\right)$ :

$\frac{\partial \Phi(p, t)}{\partial t}-\left.\frac{\partial \Phi(p, \tau)}{\partial \tau}\right|_{\tau=t_{0}}=-g \int_{t_{0}}^{t} \frac{\partial \Phi(p, \tau)}{\partial n} d \tau$

2) Integrating the above expression with respect to time for a second time, we obtain the following expression:
$\Phi(p, t)=\Phi\left(p, t_{0}\right)+\left.\frac{\partial \Phi(p, \tau)}{\partial \tau}\right|_{\tau=t_{0}}\left(t-t_{0}\right)-g \int_{t_{0}}^{t}(t-\tau) \frac{\partial \Phi(p, \tau)}{\partial n} d \tau$

Thus, $\Phi\left(p, t_{0}\right)$ and $\partial \Phi(p, \tau) /\left.\partial \tau\right|_{\tau=t_{0}}$ can be approximated by the corresponding values on the adjacent hull panel or by the value on its adjacent panel in the downstream. Similar procedure is followed for reference points and panels being at some time instant upfront the hull, then for some period of time being occupied by the hull, until the ship hull passes by.

\section{Hydrodynamic forces and motion simulation}

The unsteady pressure is given by Bernoulli's equation:

$p=-\rho\left(\frac{\partial \Phi}{\partial t}+\frac{1}{2} \nabla \Phi \cdot \nabla \Phi\right)$

Integrating this equation over the wetted surface $S_{b}$ we will obtain the hydrodynamic forces acting on the body:

$\vec{F}=-\rho \iint_{S_{b}}\left(\frac{\partial \Phi}{\partial t}+\frac{1}{2} \nabla \Phi \cdot \nabla \Phi\right) \vec{n} d s$

Here, the first term is evaluated as:

$\frac{\partial \Phi}{\partial t}=\frac{D \Phi}{D t}-\vec{v} \cdot \nabla \Phi \approx \frac{\Phi(t)-\Phi(t-\Delta t)}{\Delta t}-U_{0} \frac{\partial \Phi}{\partial x}$

For simulating linear ship motions under the excitement of small amplitude waves, all the force terms in the motion equation will be calculated linearly. For large ship motion, the Froude-Krylov and restoring forces will be calculated exactly up to the undisturbed incident wavy surface and transferred into the motion equations while the hydrodynamic forces are calculated up to the mean wetted surface. In the head-seas case, as the ship is port/starboard symmetric, there will be only heave and pitch motions, neglecting the impact of surge motions. Then the motion equation becomes:

$m \dot{v}_{3}=F_{3} \quad I_{2} \dot{\omega}_{2}=M_{2}$

It is important to notice that the rotational equation is herein formulated in the body-fixed coordinate system; $\dot{v}_{3}$ and $\dot{\omega}_{2}$ are the linear vertical and angular velocity of the mass center about the $y$-axis in the body-fixed coordinate system. They are transferred to the earth-fixed coordinate system through time dependent transformation matrices (D. Spanos, 2002).

\section{NUMERICAL IMPLEMENTATION OF PRESENT METHOD}

The boundary element method is herein used to solve the set potential theory problem numerically. The distribution of 
the potential over each panel is assumed constant. For the motion problem, we use in general about 300 panels to discretize ship's surface, 400 panels on the control surface, and 3000 panels on the free surface. When addressing more refined nonlinear effects (like higher order forces, added resistance etc.) are investigated, more panels on the hull are needed. The free surface panelization is restricted between the body boundary and a control surface, which forms the border of the inner and outer domains. The extent of the discretized free surface depends on the wave length, ship speed, and target simulating time. Typically the size of the inner domain is $(5 L-15 L) \times(B / 2+\lambda) \times \lambda / 2$ for simulating 15 20 wave periods with moderate ship speed. A time domain procedure is implemented for motion simulation purpose, where the iterative scheme is used to obtain converged motion results. The flowchart of the numerical procedure and implemented code is shown in Fig. 3.

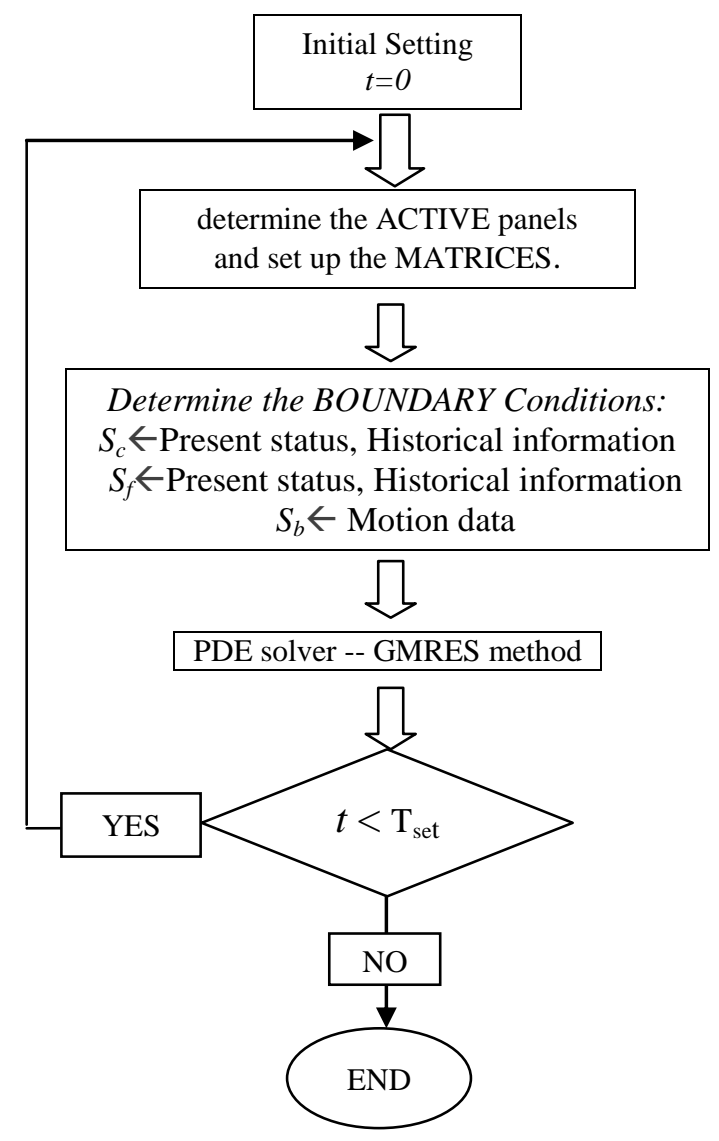

Fig. 3 Flowchart of the HYBRID method program.

\section{Special consideration on the time varying free surface grid}

As the ship is advancing through water, the intersection between the hull and the free surface will be changing over the time accordingly. This will result to a time-varying freesurface and of the corresponding numerical grid. For the panels on the free-surface, which are crossed or occupied by the moving hull, special attention is needed, in line with the deliberations in the free surface condition.

The free-surface is panelized into four zones, as shown in Fig. 4. Zone $I, I I$ and $I I I$ will not be disturbed by the moving hull so that the panelization is fixed. There are, however, two types of panelization created in Zone $I V$, namely the so-called Parent Panel System and the Sub Panel System in the frame of a Chimera grid technique (Nigel et al., 1999). For the area close to the moving hull, Sub Panels will be set as active. Far from the hull, but in Zone $I V$, Parent Panels will be set as active. There will be an information/data exchange between two sets of panels.

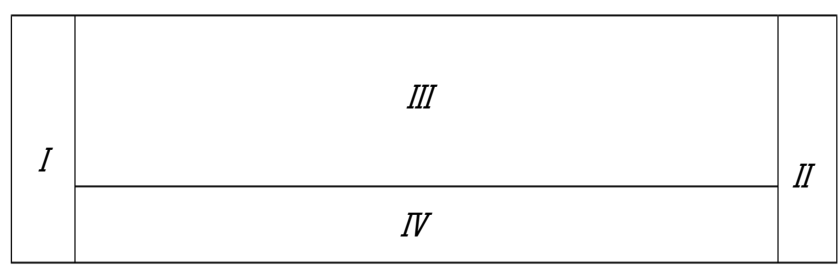

Fig. 4 Overview of the different zones of free-surface panelization.

For the free-surface condition of the parent panels, if one is marked as 0 , which means passive, so that we skip this panel; if it is marked as 1 and located behind of the hull, then we compute the free surface condition of every sub panel and store the average value; if it is marked as 1 and is located ahead of the hull, then we compute the free surface condition directly.

Likewise, for the free-surface condition of sub panels, if one is marked as 0 , it is passive so that simply we skip this panel; if it is marked as 1 and located behind the hull, then we compute the free surface condition by using their initial conditions when they became active and historical information since then; if it is marked as 1 and located ahead of the hull, then we compute the free surface condition by using their own historical information in recent steps and their parent panel's historical information in previous steps. Once the influence matrices and free surface condition are determined, equation (17) can be solved.

\section{Discretization of integral equations}

The discretized form of the linearized free-surface condition, Equation (7), is:

$$
\Phi_{j}^{M}=-g(\Delta t)^{2} \sum_{i=1}^{I-1}(I-i) \frac{\partial \Phi_{j}^{m}}{\partial n_{j}}
$$

Here $\mathrm{j}$ is the free-surface panel index, $(I-i) \Delta t$ denote the time difference from historical moment $\tau$.

The discretized form of the integral equation for the velocity potential (2) in the outer domain is:

$\sum_{j=1}^{N^{C}} A_{i j} \Phi_{j}^{M}=M e m o_{i}+\sum_{j=1}^{N^{C}} B_{i j} \Phi_{n j}^{M} \quad\left(i=1,2, \ldots, N^{c}\right)$ 
$A_{i j}=\left\{\begin{array}{cc}2 \pi+\iint_{\Delta s_{j}^{v}} \frac{\partial}{\partial n_{j}}\left(-\frac{1}{r_{i j}}\right) d s_{j} & i=j \\ \iint_{\Delta s_{j}^{u}} \frac{\partial}{\partial n_{j}}\left(\frac{1}{r_{i j}}-\frac{1}{r_{i j}}\right) d s_{j} & i \neq j\end{array}\right.$

$B_{i j}=\iint_{\Delta s_{j}^{M}}\left(\frac{1}{r_{i j}}-\frac{1}{r_{i j}}\right) d s_{j}$

$M e m o_{i}=\Delta t \sum_{m=1}^{M-1} \varepsilon_{m} \sum_{j=1}^{N^{c}}\left(\Phi_{n j}^{m} \iint_{\Delta s_{j}^{m}} \tilde{G}_{j}^{m} d s_{j}-\Phi_{j}^{m} \iint_{\Delta s_{j}^{m}} \tilde{G}_{n j}^{m} d s_{j}\right)$

Thus we have

$\Phi_{j}^{M}=\left[A_{i j}\right]^{-1}\left[M e m o_{i}+\sum_{j=1}^{N^{c}} B_{i j} \Phi_{n j}^{M}\right]\left(i=1,2, \ldots, N^{c}\right)$

The discretized form of the integral equation for the velocity potential (1) in the inner domain is:

$$
\begin{gathered}
\sum_{j=1}^{N^{c}+N^{f}+N^{b}}\left[C_{i j}(t) \Phi_{j}^{M}-D_{i j}(t) \Phi_{n j}^{M}\right]=0 \\
\left(i=1,2,3, \ldots, N^{c}+N^{f}+N^{b}\right) \\
C_{i j}(t)=\left\{\begin{array}{cc}
2 \pi & i=j \\
\iint_{\Delta s_{j}} \frac{\partial}{\partial n_{j}}\left(\frac{1}{r_{i j}}\right) d s_{j} & i \neq j
\end{array} \quad D_{i j}(t)=\iint_{\Delta s_{j}} \frac{1}{r_{i j}} d s ;\right.
\end{gathered}
$$

$N^{c}, N^{f}$ and $N^{b}$ denote the panel numbers on control surface, free surface and mean wetted body surface respectively, noting that the free surface and body surface are time-varying. Substituting the results of equation (15) into equation (16), using the free-surface condition (13) and taking into account the matching condition ( 3 ), we obtain the final equation, to be solved for the velocity potential in the entire fluid domain:

$$
\begin{gathered}
\sum_{j, k=1}^{N^{c}}\left\{-C_{i j}\left[A_{j k}\right]^{-1}\left[B_{k j}\right]-D_{i j}\right\} \Phi_{n j}^{M}+\sum_{j=N^{c}+1}^{N^{c}+N^{f}}\left(-D_{i j} \Phi_{n j}^{M}\right) \\
+\sum_{j=N^{c}+N^{f}+1}^{N^{c}+N_{i j}^{f}+N^{b}} \Phi_{j}^{M} \\
=-\sum_{j=1}^{N^{c}} C_{i j}\left\{\left[A_{i j}\right]^{-1}\left[\text { Memo }_{j}\right]_{o}\right\}-\sum_{j=N^{c}+1}^{N^{c}+N^{f}} C_{i j} \Phi_{j}^{M} \\
+\sum_{j=N^{c}+N^{f}+1} D_{i j} \Phi_{n}^{M}
\end{gathered}
$$

Equation (17) has a large influence matrix, the size of which is determined by the total number of panels on the free surface, body boundary and control surface (typically: a 4000 by 4000 matrix). In consideration of the matrix's size and structure, the Generalized Minimum Residual method (GMRES) is chosen to solve the equation. Solving this set of equations, we obtain the velocity potential and its normal derivatives on the control surface, free-surface and body surface. From the velocity potential on the body surface, the hydrodynamic pressure is calculated, the integration of which over the wetted body surface leads to the hydrodynamic forces and moments and eventually to the motion and loads responses, following the deliberations in the hydrodynamic forces and motion simulation

\section{RESULTS AND DISCUSSION}

The developed hybrid method has been numerically implemented in a computer code in the frame of the $\mathrm{PhD}$ work of the first author at the Ship Design Laboratory of NTUA. Some typical results of application are presented and discussed in the following.

\section{Steady problem of a Wigley hull}

In a first validation-case study, the present method is applied to the steady free-surface problem, namely the ship advancing at constant forward speed in calm water; herein, the calculated wave making resistance of a standard Wigley hull is presented and discussed. The studied Wigley hull is defined as $y / B=\left[1-(2 x / L)^{2}\right]\left[1-(z / H)^{2}\right]$, where $2 B / L=0.1$ and $H / L=0.0625$. The Froude number is denoted by $F n=U / \sqrt{g L}$. In the shown example, the panelization used in the computation includes in total about 3000 panels (Fig. 5).

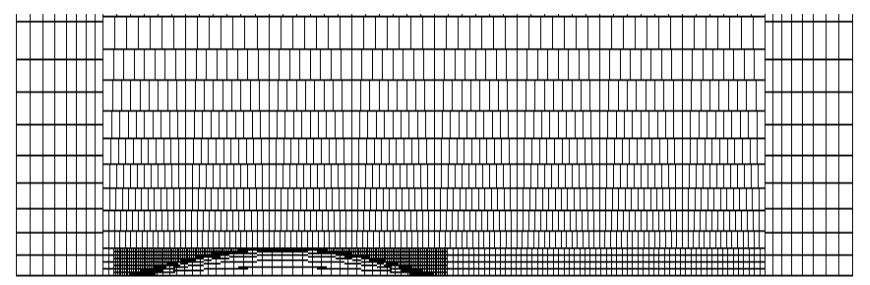

Fig. 5 Example of runtime panelization.

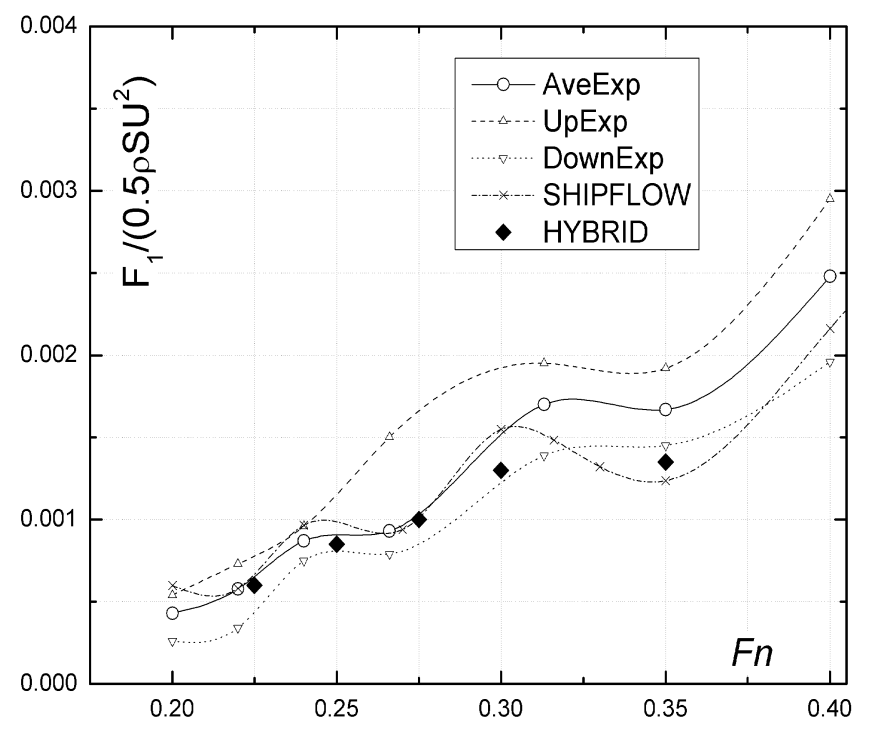

Fig. 6 Wave making resistance of a Wigley hull, without sinkage/trim correction. 
Fig. 6 shows the results for the wave making resistance of the studied Wigley hull, without sinkage/trim correction. The comparison has been made with available experimental data (Chen et al., 1983) and corresponding SHIPFLOW results (SHIPFLOW, 2005). A good agreement is observed, both with respect to the magnitude and the hump/hollow trend.

\section{Diffraction problem of $\mathrm{S} 175$ hull at $F \boldsymbol{n}=\mathbf{0 . 2 7 5}$}

The diffraction problem of ITTC S175 hull has been studied by using the present method. Fig. 7 shows the wave exciting force/moment results compared with the results from the 3D frequency domain panel code NEWDRIFT (Papanikolaou et al., 1985, 1992).

The agreement is overall very good, though the results from present method are slightly lower than those from NEWDRIFT. It should be noted that the NEWDRIFT code is based on the zero-frequency Green function method and forward speed effects are taken into account in an approximate way via slender-body theory assumptions.
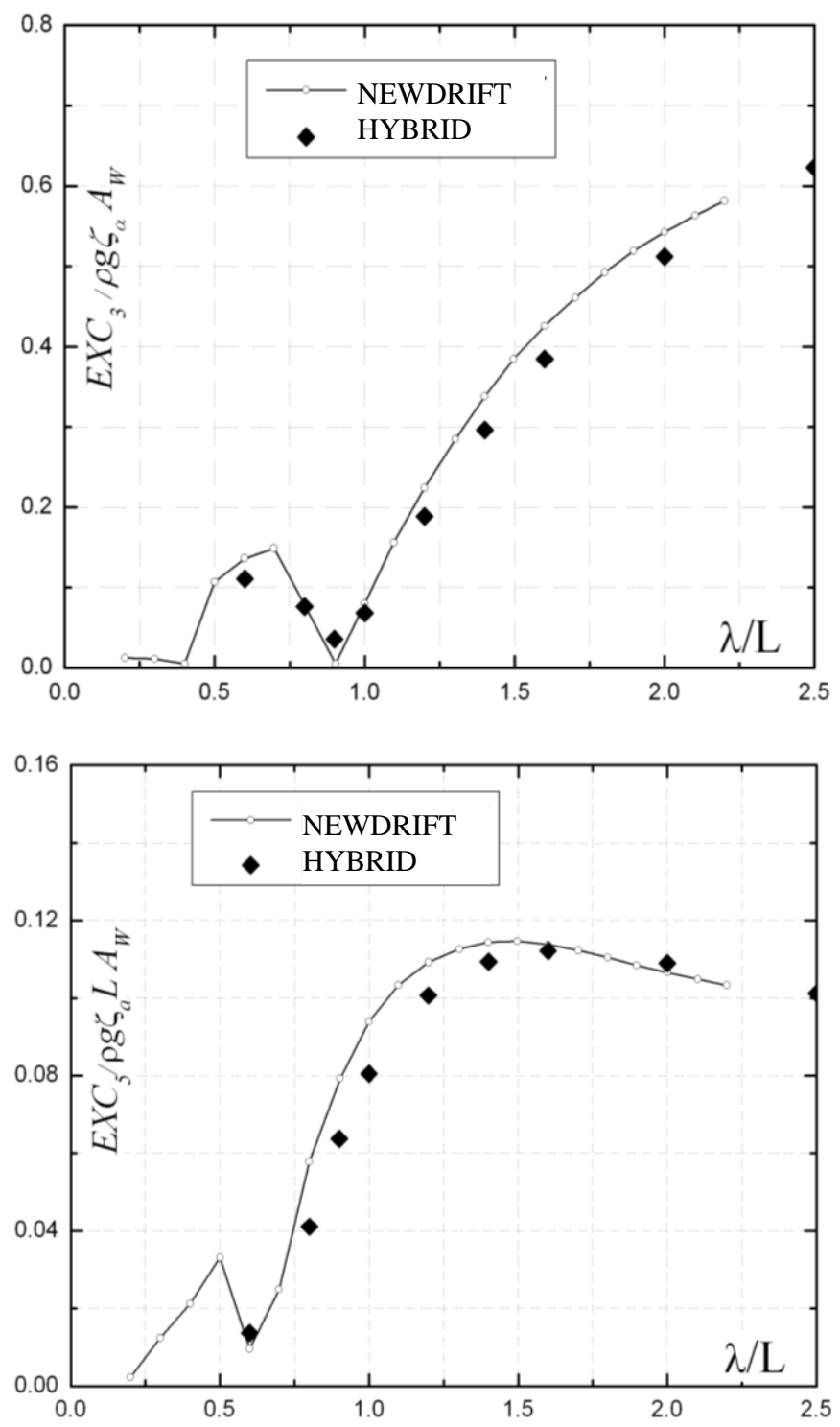

Fig. 7 Wave exciting forces of S175 hull at $F n=0.275$.
Motion simulation of $\mathrm{S175}$ hull at $\boldsymbol{F n}=\mathbf{0 . 2 7 5}$ and varying wave conditions

The present method has been adapted to simulate ship motions, both by a linear and nonlinear scheme. The nonlinearity here includes contributions from the FroudeKrylov and restoring forces/moments. Fig. 8 shows the comparison of results of the present hybrid method with available experimental data (ITTC, 1978) and results of the 3D frequency-domain panel code NEWDRIFT. For the nonlinear simulations, the incident wave steepness was varied systematically, namely $\zeta_{\alpha} / \lambda=0.01,0.02$ and 0.04 respectively.

For the linear simulation, the present method gives some results lower than NEWDRIFT and closer to the experimental data. For the nonlinear simulations, the motion amplitudes decrease gradually as the wave steepness increases. This is physically meaningful, considering the increased damping and restoring due to the above water flared hull form of S175.
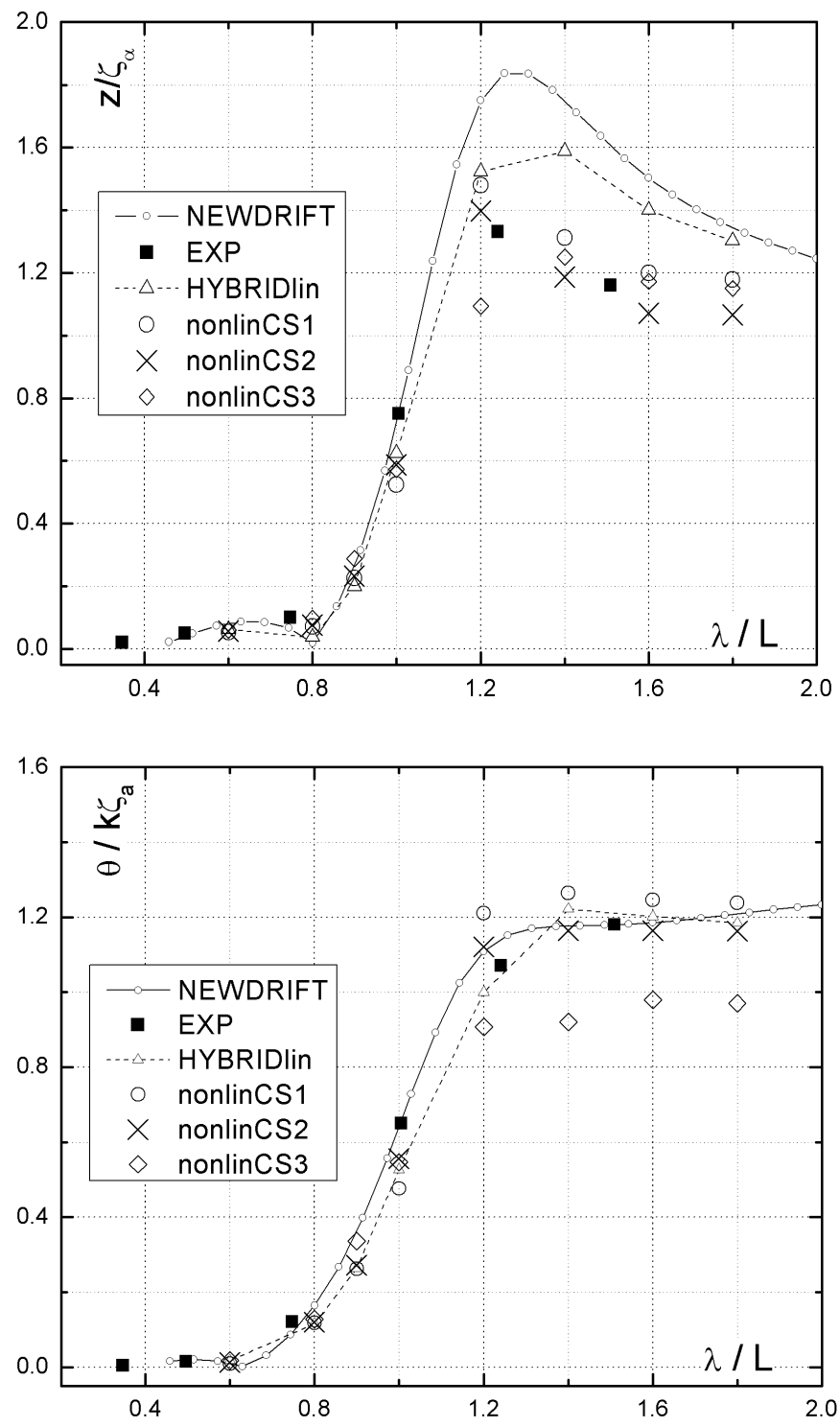

Fig. 8 Motion amplitude of S175 hull at $F n=0.275$. 
On the other side, for $\zeta_{\alpha} / \lambda=0.04$ the resulting pitch motion is much lower than experimental results. It should be noted that the wave steepness corresponding to the experimental data is not known for a better assessment.

\section{Added resistance of $\mathrm{S175}$ hull at $\mathrm{Fn}=\mathbf{0 . 2 7 5}$}

The added resistance of the ITTC S175 ship in head seas was also calculated by using the data obtained from the present method. The direct way is to calculate both the wave making resistance in calm water and resistance in waves and then get the difference between them; however, here Maruo's far-field theory (1963) is adapted to the added resistance prediction. The results agree very well with the experimental data (Takahashi, 1988). Results from NEWDRIFT and short wave range corrections based on Faltinsen's formula (1980) and Kuroda and Tsujimoto's formula (2008) respectively are also shown in Fig. 9 for comparison (noted as SW1 and SW2 respectively).

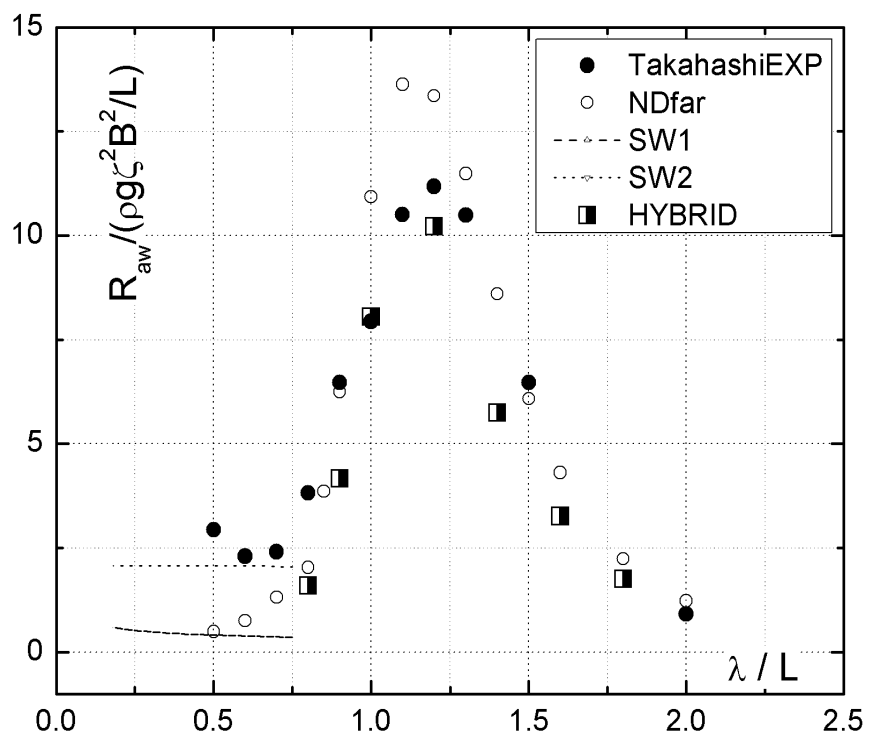

Fig. 9 Added resistance of S175 ship at $F n=0.275$.

\section{CONCLUSIONS}

A new time domain hybrid method for simulating large amplitude motions of ships advancing with constant forward speed in waves is presented. The method is hybrid in the way of combining a time-domain transient Green function method and a Rankine source method. The method has been numerically implemented in a newly developed computer code. Preliminary validation results include the estimation of the wave making resistance and wave exciting forces, the nonlinear simulation of ship motions and the estimation of added resistance in head sea conditions. Comparisons of results were made mainly with results of code NEWDRIFT, which is based on 3D panel, frequency domain theory and available experimental data. Good agreement is observed for all case studies, which indicates that the present formulation and numerical scheme are correct and working properly. In future work this method should be applied to irregular incidental wave cases and oblique seas conditions.

\section{ACKNOWLEDGEMENTS}

The presented work is part of the $\mathrm{PhD}$ work of the first author; the financial support of NTUA-SDL and the continuous guidance by the second author are highly appreciated. The initial part of this work was financially supported by the EU funded ASIA LINK programme (ASI/B7-301/98/679-044 (072433)) and thereafter by a stipend of NTUA's Research Committee. Prof. W. Duan (Harbin Engineering University) and Dr. D. Spanos (NTUASDL) have contributed to this work through many discussions, which proved very important for the development of the present method, thus their support is also appreciated.

\section{REFERENCES}

Chen, C.Y. and Noblesse, F., 1983. Comparison between theoretical predictions of wave resistance and experimental data for the Wigley hull. Journal of Ship Research.Vol.27, pp.215-226.

Cummins, W.E., 1962. The impulsive response function and ship motions, Schiffstechnik, Vol.9, pp.124-135.

Duan, W. and Dai, Y., 1999. Time-domain calculation of hydrodynamic forces on ships with large flare. International Shipbuilding Progress, Vol.46, pp.223-232.

Faltinsen, O. M. Minsaas, K. J. Liapis, N. and Skjørdal, S. O., 1980. Prediction of resistance and propulsion of a ship in a seaway. Proc. 13th Symposium on Naval Hydrodynamics, Tokyo, Japan, pp. 505-529.

Finkelstein, A., 1957. The initial value problem for transient water waves. Communications on Pure and Appled. Mathematics, Vol.10, pp.511-522.

ITTC Seakeeping Committee, 1978. Comparison of results obtained with compute programs to predict ship motions in six-degrees-of-freedom and associated responses. Proc. 15th ITTC, pp.79-92.

Kataoka, S. and Iwashita, H., 2004. Estimations of hydrodynamic forces acting on ships advancing in the calm water and waves by a time-domain hybrid method. Journal of the Society of Naval Architects of Japan, Vol.196, pp.123-138.

Kataoka, S. and Iwashita, H., 2005. Seakeeping estimations of ships by a time-domain hybrid method, International. RIAM Symposium, Kyushu, Japan.

Kuroda, M. Tsujimoto, M. and Fujiwara, T., 2008. Investigation on components of added resistance in short waves, Journal of the Japan Society of Naval Architects and Ocean Engineers, Vol. 8, pp. 171-176. 
Liu, S. Papanikolaou, A. and Duan, W., 2006. A time domain numerical simulation method for nonlinear ship motions. Journal of Harbin Engineering University, Vol.27, pp. 177-185.

Liu, S. and Papanikolaou, A., 2009. A time-domain hybrid method for calculating hydrodynamic forces on ships in waves. 13th Inter. Congress of the Inter. Maritime Association of the Mediterranean, Istanbul, Turkey.

Maruo, H., 1963. Resistance in waves, 60th anniversary Series. The Society of Naval Architects of Japan, 8, pp.67-102.

Nigel, P. Weatherill. Bharat, K. Soni. and Joe, F. Thompson., 1999. Handbook of Grid Generation, CRC Press.

Papanikolaou, A., 1985. On integral-equation-methods for the evaluation of motions and loads of arbitrary bodies in waves. Journal Ingenieur - Archiv., Vol.55, pp.17-29.

Papanikolaou, A. and Schellin, Th., 1992. A threedimensional panel method for motions and loads of ships with forward speed in waves. Journal Schiffstechnik, Vol. 39, pp.145-156.

SHIPFLOW, 2005., SHIPFLOW 3.0 examples manual, http://www.flowtech.se/
Spanos, D., 2002. Simulation of the motions of a ship after flooding in waves and investigation of the survivability of ROPAX ships. Doctoral Thesis, National Technical University of Athens, Greece.

Takahashi, T., 1988. A practical prediction method for added resistance of a ship in waves and the direction of its application to hull form design. Trans. Of the West Japan Society of Naval Architects, Vol.75, pp.75-95.

Tsujimoto, M. Shibata, K. and Kuroda, M., 2008. A practical correction method for added resistance in waves, Journal of the Japan Society of Naval Architects and Ocean Engineers, Vol. 8, pp. 177-184.

Wang, J., 2003. Numerical simulation of the linear freesurface condition. Master Thesis, Harbin Engineering University, China.

Yasukawa, H., 2003. Application of a 3-D Time Domain Panel Method to Ship Seakeeping Problems. 24th Symposium on Naval Hydrodynamics, pp.376-392.

Zhang, S. Lin, W.M. and Weems, K., 1998. A hybrid boundary element method for non-wall-sided bodies with or without forward speed. 13th IWWWFB, pp. 179-182. 